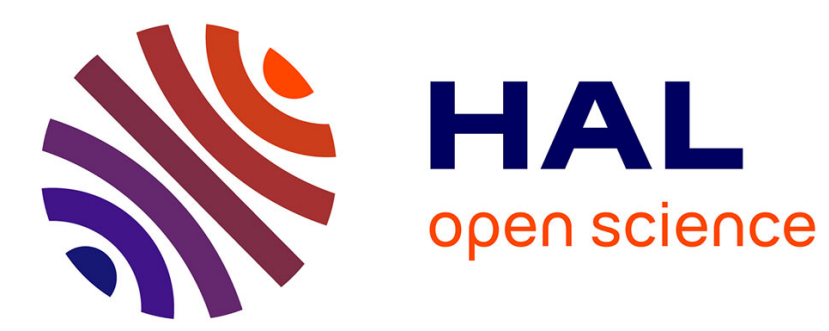

\title{
PROPAGATING MODES OF A HETEROGENEOUS, MACRO-HOMOGENEOUS CONTINUUM
}

\author{
J. Korringa
}

\section{To cite this version:}

J. Korringa. PROPAGATING MODES OF A HETEROGENEOUS, MACRO-HOMOGENEOUS CONTINUUM. Journal de Physique Colloques, 1972, 33 (C6), pp.C6-117-C6-122. 10.1051/jphyscol:1972626 . jpa-00215144

HAL Id: jpa-00215144

https://hal.science/jpa-00215144

Submitted on 1 Jan 1972

HAL is a multi-disciplinary open access archive for the deposit and dissemination of scientific research documents, whether they are published or not. The documents may come from teaching and research institutions in France or abroad, or from public or private research centers.
L'archive ouverte pluridisciplinaire HAL, est destinée au dépôt et à la diffusion de documents scientifiques de niveau recherche, publiés ou non, émanant des établissements d'enseignement et de recherche français ou étrangers, des laboratoires publics ou privés. 


\title{
PROPAGATING MODES OF A HETEROGENEOUS, MACRO-HOMOGENEOUS CONTINUUM
}

\author{
J. KORRINGA
}

Department of Physics,

The Ohio State University, Columbus, Ohio 43210, U.S. A

\begin{abstract}
Résumé. - Une méthode est développée pour calculer la dispersion des "phonons 》 dans un milieu hétérogène et macro-homogène. On définit une solution de l'équation d'ondes élastiques à l'intérieur d'un volume $V$, laquelle est périodique dans le temps et satisfait des conditions limites sur la surface $\Sigma$ de $V$ comme s'il s'agissait d'une onde plane. Le problème est mis sous forme d'une équation intégrale à l'aide de la fonction de Green d'un milieu « modèle » qui a une densité et un tenseur d'élasticité constants. Elle est mise dans une forme telle que la limite $V \rightarrow \infty$ peut être effectuée. Un milieu « effectif » est défini en termes de la solution de cette équation intégrale, en prenant la moyenne dans l'espace de l'amplitude des ondes élastiques. Ceci donne une équation implicite pour la relation de dispersion. L'équation intégrale est approximée en utilisant une méthode d'inclusion par laquelle l'amplitude moyenne de l'onde est obtenue comme moyenne d'ensemble de solutions dans une petite région, incluse dans le milieu modèle. Les erreurs dues à cette méthode d'inclusion sont réduites au minimum en imposant que le milieu modèle et le milieu effectif approximatif soient identiques. Ceci donne une méthode itérative et self-consistante qui peut être approximée avec des méthodes numériques sans introduire de fonctions de corrélation.
\end{abstract}

\begin{abstract}
A method is developed to calculate the dispersion of propagating elastic modes in heterogeneous, macro-homogeneous media. A time-periodic solution of the wave equation is defined inside a volume $V$ by imposing a plane-wave character on the boundary $\Sigma$ completely surrounding $V$. The equation is transformed into an integral equation with use of a Green's function for a " model-medium ", with constant elastic coefficients and density. It is brought in such a form, that the limit $V \rightarrow \infty$ can be taken. A uniform " effective medium " is defined, in terms of the solution of this integral equation, by taking the space average of the wave equation. This gives an implicit equation for the dispersion relation. An approximation analogous to the CPA is obtained. The integral equation is approximated in terms of an imbedding method, through which the average wave amplitude is obtained as an ensemble average of solutions for small neighbourhoods embedded in the model medium. By imposing the condition that the modelmedium and the effective medium are identical, the errors of imbedding are minimized. This yields a self-consistent iterative procedure. The case of long wavelength or low density contrast leads to simplified equations analogous to those for the static limit. This case can be approximated with use of correlation functions, as shown elsewhere.
\end{abstract}

1. Introduction. - The following is an attempt to define propagating modes of a macro-homogeneous elastic continuum - in contradistinction to "local" modes. The aim is to arrive at a dispersion relation for such modes, $\omega(k)$, using a labeling by a wavevector $k$, as for plane waves. It is my contention that a distinction between propagating modes and local modes is meaningful for excitations of "low » frequencies, where the former are a vast majority. Thus I am aiming at a theory which, while valid at low frequencies, may lead to divergences at higher frequency. But I want no restrictions of the properties of the medium - i. e., no assumption of "weak scattering " by impurities, or low concentration of scattering sites.

Precisely what constitutes low frequencies is better left unsaid at this stage, but I presume that my method breaks down when the wavelength is of the order of the size of the grains or cavities of the medium, as modes of this wavelength will not propagate.

Except for the case of homogeneous media, planewave like modes will not be strictly stationary, because there is no translation-invariance or invariance under any group of related transformations. One must therefore expect a complex dispersion relation $\omega(k)$ which I prefer to express as a complex function of the real variables $k\left(k_{1}, k_{2}, k_{3}\right)$.

The literature on wave propagation these days is dominated by such problems as the bandstructure of random alloys, where the wave equation is of the Schrödinger type. Progress is based on the use of the resolvent [1] and of the coherent potential approximation (CPA) [2], which gives the « best " approximation in terms of composition only, i. e., neglecting specific effects of structure. My reasons for not using this method here are several. Firstly, it is unnatural. 
The classical wave equation requires a transformation involving the square root of the mass-density, for the method to apply, and this makes the problem look harder than its quantum-mechanical counterpart, where in reality it is much easier. Secondly, for many classical problems the CPA is totally inadequate and while proposals for generalisation have not been lacking [3], difficulties and ambiguities abound. I attribute at least part of these difficulties to the fact that in the resolvent method the contribution of propagating and local modes to the density of states is inseparable. I imagine that these two types of modes depend quite differently on local groupings of scatterers, and I think that this causes difficulties with almost any attempt to improve on the CPA. Thirdly, by "falling back» on a method based directly on the wave equation, such as the one to be outlined presently, one may lose some advantages in formal calculations, but regains insight into what is going on, which is useful when it comes to making approximations. Fourthly, this paper deals exclusively with propagating modes, so that the local modes, which I hope to have eliminated correctly, do not need to be calculated separately.

With these apologies, I will let the equations speak for themselves.

2. Derivation of the fundamental integral equation. - 2.1 WAVE EQUATION AND BOUNDARY CONDITIONS. - The wave equation for modes with frequency $\omega$ in a medium with elastic tensor $C(x)$ and massdensity $\rho(x)$, filling a volume $V$ bounded by $\Sigma$ is

$$
\begin{aligned}
\mathfrak{L}(x) u_{i}(x) \equiv \partial_{j} C_{i j p q}(x) \partial_{q} u_{p}(x)+ & +\rho(x) \omega^{2} u_{i}(x)=0, \quad x \in V .
\end{aligned}
$$

As boundary condition I use

$$
u_{p}(\hat{x})=\hat{u}_{p}^{0} \mathrm{e}^{i k \hat{x}}, \quad \hat{x} \in \Sigma,
$$

where $k$ is any real vector and $\hat{u}^{0}$ a constant vector. Simultaneously, I consider the wave equation in a uniform medium, which I call "model-medium":

$$
\mathfrak{L}^{0} u_{i}^{0}(x) \equiv \partial_{j} C_{i j p q}^{0} \partial_{q} u_{p}^{0}(x)+\rho^{0} \omega^{2} u_{i}^{0}(x)=0
$$

with boundary conditions as in eq. (2) :

$$
u_{p}^{0}(\hat{x})=\widehat{u}_{p}^{0} \mathrm{e}^{i k \hat{x}}, \quad x \in \Sigma .
$$

Eq. (4) will also hold in the interior of $V$ if and only if the values of $C^{0}, \rho^{0}, \omega^{2}$ and $k$ are suitably related and the amplitude $\hat{u}_{p}^{0}$ is along one of the possible polarisation vectors for these values. Ultimately, only this situation is of importance, but the following derivation is general.

2.2 Green's THEOREM. - Let $G_{i l}\left(x, x^{\prime}\right)$ be the radiative Green's function of (3), satisfying

$$
\begin{gathered}
\mathfrak{L}^{0} G_{i l}\left(x, x^{\prime}\right)=-\delta_{i l} \delta^{3}\left(x-x^{\prime}\right), \\
G\left(x, x^{\prime}\right) \sim\left|x-x^{\prime}\right|^{-1}, \quad\left|x-x^{\prime}\right| \rightarrow \infty .
\end{gathered}
$$

For example, for an isotropic medium,

$$
C_{i j p q}^{0}=\lambda^{0} \delta_{i j} \delta_{p q}+\mu^{0}\left(\delta_{i p} \delta_{j q}+\delta_{i q} \delta_{j p}\right),
$$

one has

$$
\begin{array}{r}
G_{i l}\left(x, x^{\prime}\right)=\frac{1}{4 \pi \rho^{0} \omega^{2}}\left[\partial_{i} \partial_{l} R^{-1}\left(\mathrm{e}^{-i K_{s} R}-\mathrm{e}^{-i K_{p} R}\right)+\right. \\
\left.+K_{s}^{2} \delta_{i l} R^{-1} \mathrm{e}^{-i K_{s} R}\right]
\end{array}
$$

where

$$
R^{2}=\left(x_{i}-x_{i}^{\prime}\right)\left(x_{i}-x_{i}^{\prime}\right),
$$

$K_{p}^{2}=\omega^{2} \rho^{0} /\left(\lambda^{0}+2 \mu^{0}\right), \quad K_{s}^{2}=\omega^{2} \rho^{0} / \mu^{0}, \quad \operatorname{Im} K \leqslant 0$.

For any symmetry, one has

$$
G_{i l}\left(x-x^{\prime}\right)=G_{i l}\left(x^{\prime}-x\right)=G_{l i}\left(x^{\prime}-x\right) .
$$

Green's theorem in the form

$$
\begin{array}{r}
\int_{V} d^{3} x^{\prime}\left[U_{i}\left(x^{\prime}\right) \mathcal{L}^{0} V_{i}\left(x^{\prime}\right)-V_{i}\left(x^{\prime}\right) \mathcal{L}^{0} U_{i}\left(x^{\prime}\right)\right]= \\
=\int_{\Sigma} d^{2} \hat{x}^{\prime}\left[U_{i}\left(\hat{x}^{\prime}\right) C_{i j p q}^{0} n_{j} \partial_{q}^{\prime} V_{p}\left(\hat{x}^{\prime}\right)-\right. \\
\left.-V_{i}\left(\hat{x}^{\prime}\right) C_{i j p q}^{0} n_{j} \partial_{q}^{\prime} U_{p}\left(\hat{x}^{\prime}\right)\right]
\end{array}
$$

is now used with

$$
\begin{gathered}
V_{p}\left(x^{\prime}\right)=u_{p}\left(x^{\prime}\right)-u_{p}^{0}\left(x^{\prime}\right), \\
U_{i}\left(x^{\prime}\right)=G_{i l}\left(x^{\prime}, x\right), \quad l=1,2,3,
\end{gathered}
$$

where $u_{p}\left(x^{\prime}\right)$ is the solution of (1) and (2), $u_{p}^{0}\left(x^{\prime}\right)$ the solution of (3) and (4). With substitution from (1) and (3), and using (2) and (4), this gives

$$
\begin{aligned}
& u_{l}(x)-u_{l}^{0}(x)+ \\
& \quad+\int_{V} d^{3} x^{\prime} G_{i l}\left(x^{\prime}, x\right) \hat{\partial}_{j}^{\prime}\left[C_{i j p q}\left(x^{\prime}\right)-C_{i j p q}^{0}\right] \partial_{q}^{\prime} u_{p}\left(x^{\prime}\right) \\
& +\omega^{2} \int_{V} d^{3} x^{\prime} G_{p l}\left(x^{\prime}, x\right)\left[\rho\left(x^{\prime}\right)-\rho^{0}\right] u_{p}\left(x^{\prime}\right)= \\
& \quad=\int_{\Sigma} d^{2} \hat{x}^{\prime} G_{i l}\left(\hat{x}^{\prime}, x\right) C_{i j p q}^{0} n_{j}^{\prime} \partial_{q}^{\prime}\left[u_{p}\left(\hat{x}^{\prime}\right)-u_{p}^{0}\left(\hat{x}^{\prime}\right)\right] .
\end{aligned}
$$

2.3 Gauss' THEOREM. - As an essential step in my method, the first integral in (13) is now transformed using Gauss' theorem,

$$
\int_{V} d^{3} x\left(U \partial_{j} V+V \partial_{j} U\right)=\int_{\Sigma} d^{2} \hat{x} U V n_{j}
$$

Let

$$
\varepsilon_{p q}(x)=\partial_{(q} u_{p)}(x) \equiv \frac{1}{2}\left(\partial_{q} u_{p}(x)+\partial_{p} u_{q}(x)\right)
$$

and define

$$
p_{i j}(x)=\left[C_{i j p q}(x)-C_{i j p q}^{0}\right] \varepsilon_{p q}(x),
$$


which is equal to the expression in the first integrand because $C_{i j p q}$ and $C_{i j p q}^{0}$ are symmetric in $p$, q. One has $p_{i j}=p_{j i}$ because $C_{i j p q}$ and $C_{i j p q}^{0}$ are symmetric in $i, j$. Application of (14) introduces a more singular kernal,

$$
S_{l i j}\left(x^{\prime}, x\right)=\partial_{(j} G_{i) l}\left(x^{\prime}, x\right)
$$

Excluding a small volume $v$ with surface $\sigma$ around the point $x^{\prime}=x$, and using the normal on $\sigma$ and $\Sigma$ away from the singular point, one has

$$
\begin{aligned}
\int_{V-v} d^{3} x^{\prime} & G_{i l}\left(x^{\prime}, x\right) \partial_{j}^{\prime} p_{i j}\left(x^{\prime}\right)= \\
= & \int_{V-v} d^{3} x^{\prime} S_{l i j}\left(x^{\prime}, x\right) p_{i j}\left(x^{\prime}\right)+ \\
& +\int_{\Sigma-\sigma} d^{2} \hat{x}^{\prime} G_{i l}\left(\hat{x}^{\prime}, x\right) p_{i j}\left(\hat{x}^{\prime}\right) n_{j}^{\prime}
\end{aligned}
$$

These transformations bring eq. (13) into the form

$$
\begin{aligned}
u_{l}(x)- & u_{l}^{0}(x)+\int_{V-v} d^{3} x^{\prime} S_{l i j}\left(x^{\prime}, x\right) p_{i j}\left(x^{\prime}\right)+ \\
& +\int_{V=v} d^{3} x^{\prime} G_{i l}\left(x^{\prime}, x\right) \omega^{2}\left[\rho\left(x^{\prime}\right)-\rho^{0}\right] u_{i}\left(x^{\prime}\right) \\
& +\int_{\sigma} d^{2} \hat{x}^{\prime} G_{i l}\left(\hat{x}^{\prime}, x\right) p_{i j}\left(\hat{x}^{\prime}\right) n_{j}= \\
= & \int_{\Sigma} d^{2} \hat{x}^{\prime} G_{i l}\left(\hat{x}^{\prime}, x\right) \times \\
& \times\left\{C_{i j p q}^{0} \hat{\sigma}_{q}^{\prime}\left[u_{p}\left(\hat{x}^{\prime}\right)-u_{p}^{0}\left(\hat{x}^{\prime}\right)\right]+p_{i j}\left(\hat{x}^{\prime}\right)\right\} n_{j} .
\end{aligned}
$$

2.4 FURTHER TRANSFORMATIONS. - The next step is to make eq. (19) into an equation for the strain $\varepsilon_{k l}(x)$, defined by eq. (15), by taking the derivative $\partial_{k}$ and symmetrizing. The index $k$ should not be confused with the wave vector. Owing to the exclusion of the singular point, the derivative can be taken under the integral sign. This gives an equation just like (19), but with $u_{l}$ and $u_{l}^{0}$ in the first term replaced by $\varepsilon_{k l}$ and $\varepsilon_{k l}^{0}$, while in the integrals $G_{i l}$ is replaced by $S_{i k l}$ and $S_{l i j}$ by $T_{i t i j}$, defined by

$$
T_{k l i j}\left(x^{\prime}, x\right)=\partial_{(k} S_{l) i j}\left(x^{\prime}, x\right) .
$$

From eq. (10), it follows that $G, S$ and $T$ are symmetric in all their indices, and that $G$ and $T$ are even in $\left(x-x^{\prime}\right)$, while $S$ is odd.

In the resulting equation, the limit $v \rightarrow 0$ can be taken. The first integral becomes the principal value, and the integral over $\sigma$ can be done. Defining

$$
\lim _{v \rightarrow 0} \int_{\sigma} d^{2} \hat{x}^{\prime} S_{i k l}\left(\hat{x}^{\prime}, x\right) n_{j}=-\Gamma_{k l i j},
$$

one has

$$
\lim _{v \rightarrow 0} \int_{\sigma} d^{2} \hat{x}^{\prime} S_{i k l}\left(\hat{x}^{\prime}, x\right) p_{i j}\left(\hat{x}^{\prime}\right) n_{j}=-\Gamma_{k l i j} p_{i j}(x) .
$$

The components of $\Gamma$ are constants depending on $C^{0}$, $\rho^{0}$ and $\omega$ in a known manner.

Next the limit $V \rightarrow \infty$ is required, uniformly to all sides, as for a sphere. For real $\omega$ the integral over $\Sigma$ does not go to zero and, indeed, may diverge in the limit, on account of the term in $p_{i j}$. Using the fact that a wave-like field is imposed at $\Sigma$, I resolve this dilemma by defining a constant $\ddot{p}_{i j}$ such that

$$
\begin{array}{r}
\lim _{\Sigma \rightarrow \infty} \int_{\Sigma} d^{2} \hat{x}^{\prime} S_{i k l}\left(\hat{x}^{\prime}, x\right)\left\{C_{i j p q}^{0} \partial_{q}^{\prime}\left[u_{p}\left(\hat{x}^{\prime}\right)-u_{p}^{0}\left(\hat{x}^{\prime}\right)\right]+\right. \\
\left.+p_{i j}\left(\hat{x}^{\prime}\right)-\ddot{p}_{i j} \mathrm{e}^{i k \hat{x}^{\prime}}\right\} n_{j}=0
\end{array}
$$

The term thus subtracted can also be written as

$$
\begin{gathered}
\bar{p}_{i j} \int_{\Sigma} d^{2} \hat{x}^{\prime} S_{i k l}\left(\hat{x}^{\prime}, x\right) \mathrm{e}^{i k \hat{x}^{\prime}} n_{j}=-\Gamma_{k l i j} \bar{p}_{i j} \mathrm{e}^{i k x}+ \\
+\bar{p}_{i j} \int_{V} d^{3} x^{\prime} \partial_{j}^{\prime}\left[S_{i k l}\left(x^{\prime}, x\right) \mathrm{e}^{i k x^{\prime}}\right] .
\end{gathered}
$$

With this one can take the limit $V \rightarrow \infty$, obtaining

$$
\begin{aligned}
& \varepsilon_{k l}(x)-\varepsilon_{k l}^{0}(x)+ \\
& +\int d^{3} x^{\prime}\left\{T_{k l i j}\left(x^{\prime}, x\right)\left[p_{i j}\left(x^{\prime}\right)-\bar{p}_{i j} \mathrm{e}^{i k x^{\prime}}\right]+\right. \\
& \left.+S_{i k l}\left(x^{\prime}, x\right)\left[i k_{j} \bar{p}_{i j} \mathrm{e}^{i k x^{\prime}}+\omega^{2}\left(\rho\left(x^{\prime}\right)-\rho^{0}\right) u_{i}\left(x^{\prime}\right)\right]\right\}- \\
& -\Gamma_{k l i j}\left[p_{i j}(x)-\bar{p}_{i j} \mathrm{e}^{i k x}\right]=0 .
\end{aligned}
$$

2.5 Elimination of 8 . - With use of eq. (16), $\varepsilon_{k l}(x)$ can now be eliminated from the principal term of the equation. Defining

$$
\varphi(x)=\left[\left(C(x)-C^{0}\right)^{-1}-\Gamma\right]^{-1},
$$

where the inverse $A^{-1}$ of a tensor $A$ is defined by

$$
A_{i j k l}^{-1} A_{k l p q}=\frac{1}{2}\left(\delta_{i p} \delta_{j q}+\delta_{i q} \delta_{j p}\right),
$$

it suffices to multiply eq. (25) with $\varphi$. Finally, in order to express everything in terms of wave amplitudes, I define

$$
\begin{aligned}
& \hat{p}(x)=p(x) \mathrm{e}^{-i k x}, \\
& \hat{u}(x)=u(x) \mathrm{e}^{-i k x}, \\
& \hat{\varepsilon}_{i j}^{0}=\frac{1}{2}\left(k_{i} \hat{u}_{j}^{0}+k_{j} \hat{u}_{i}^{0}\right) \\
& \widehat{T}\left(x-x^{\prime}\right)=T\left(x-x^{\prime}\right) \mathrm{e}^{i k\left(x^{\prime}-x\right)} \\
& \hat{S}\left(x-x^{\prime}\right)=S\left(x-x^{\prime}\right) \mathrm{e}^{i k\left(x^{\prime}-x\right)} .
\end{aligned}
$$


With this, the integral equation takes the form

$$
\begin{aligned}
\hat{p}_{r s}(x) & +\varphi_{r s k l}(x) \int d^{3} x^{\prime}\left\{\hat{T}_{k l i j}\left(x-x^{\prime}\right)\left(\hat{p}_{i j}\left(x^{\prime}\right)-\bar{p}_{i j}\right)+\right. \\
& \left.+\widehat{S}_{i k l}\left(x-x^{\prime}\right)\left[i k_{j} \bar{p}_{i j}+\omega^{2}\left(\rho\left(x^{\prime}\right)-\rho^{0}\right) \hat{u}_{i}\left(x^{\prime}\right)\right]\right\} \\
& +\varphi_{r s k l}(x)\left[\Gamma_{k l i j} \bar{p}_{i j}-\hat{\varepsilon}_{k l}^{0}\right]=0 .
\end{aligned}
$$

This equation is supplemented by eq. (23) for the definition of $\bar{p}_{i j}$ and eq. (16) for the relation between $p_{i j}(x)$ and $u_{i}(x)$.

2.6 Determination of $\bar{p}$. - I will now show that eq. (23) for $\bar{p}$ can be replaced with

$$
\bar{p}_{i j}=\left\langle\hat{p}_{i j}(x)>\equiv \lim _{V \rightarrow \infty} \frac{1}{V} \int_{V} \hat{p}_{i j}(x) d^{3} x\right.
$$

provided $k$ and $\omega$ satisfy the dispersion relation for propagating modes (to be calculated) of the medium $C(x), \rho(x)$ as well as of $C^{0}, \rho^{0}$.

As propagating mode I define a solution of eq. (1) and (2) such that the average strain amplitude equals the plane-wave strain amplitude $\widehat{\varepsilon}^{0}$ imposed at the boundary :

$$
<\widehat{\varepsilon}_{k l}>=\widehat{\varepsilon}_{k l}^{0},
$$

or, equivalently

$$
<\widehat{u}_{l}>=\widehat{u}_{l}^{0} .
$$

By averaging eq. (25) - after multiplication with $\mathrm{e}^{-i k x}-$ one sees that this implies

$$
<\hat{p}_{i j}>=\bar{p}_{i j}
$$

and

$$
\omega^{2}\left[<\rho(x) \hat{u_{i}}(x)>-\rho^{0}<u_{i}(x)>\right]=-i k_{j} \bar{p}_{i j} .
$$

Eq. (32) is the desired expression for $\bar{p}$. It comes as no surprise, considering the form of eq. (23). Eq. (33) can be considered as a relation for $C^{0}$ and $\rho^{0}$, to be satisfied if the medium $C^{0}, \rho^{0}$ and the medium $C(x), \rho(x)$ are to have the same dispersion relation. From the definition of $p_{i j}(x)$ one sees, after a simple transformation, that eq. (33) equates the mean values of the left hand sides of the wave eq. (1) and (3) :

$$
<\mathrm{e}^{-i k x} \mathfrak{L}(x) u_{i}(x)>=\mathrm{e}^{-i k x} \mathfrak{L}^{0} u_{i}^{0}(x) .
$$

3. Methods of solving the integral equation. 3.1 Definition of THE EFFECTIVE MEDIUM. Eq. (29) and (16) together with eq. (31)-(33) form a complicated eigenvalue problem. By introducing an «effective medium 》 this problem is decomposed in two parts. I define the effective elastic tensor $C^{*}$ and the effective density $\rho^{*}$ by the following equations :

$$
\begin{aligned}
<\mathrm{e}^{-i k x} C_{i j p q}(x) \varepsilon_{p q}(x)> & =C_{i j p q}^{*}<\hat{\varepsilon}_{p q}(x)>, \\
<\mathrm{e}^{-i k x} \rho(x) u_{i}(x)> & =\rho^{*}<\hat{u}_{i}(x)>.
\end{aligned}
$$

As a consequence one has, using eq. (31) :

$$
<\mathrm{e}^{-i k x} \mathfrak{L}(x) u_{i}(x)>=\mathrm{e}^{-i k x} \mathfrak{L}^{*} u_{i}^{0}(x),
$$

but in contrast to eq. (34) his equation is by definition satisfied for the terms in $C$ and $\rho$ separately. $C^{*}$ and $\rho^{*}$ are functions of $k$ and $\omega$.

3.2 Program for calculating $\omega(k)$. - The problem of finding the frequency $\omega$ for a given $k$ can now be formulated as follows. $C^{0}, \rho^{0}$ and $\widehat{u}_{i}^{0}$ are chosen in such a way that they satisfy the wave equation for a given $\omega$ :

$$
\mathfrak{L}^{0} \hat{u}_{i}^{0} \mathrm{e}^{i k x}=0
$$

and that $C^{0}$ refiects the symmetry of the medium. For example, $C^{0} \sim\langle C(x)\rangle, \rho^{0} \sim\langle\rho(x)\rangle$. Then $\hat{p}_{\text {rs }}(x)$ and $\hat{u}_{2}(x)$ are solved from eq. (29) and (16), keeping $\bar{p}_{i j}$ as an unknown constant. From this one calculates $C^{*}$ and $\rho^{*}$, using eq. (35) and (36), the former of which can be written as

$$
C_{i j p q}^{*} \hat{\varepsilon}_{p q}^{0}=C_{i j p q}^{0} \hat{\varepsilon}_{p q}^{0}+<\hat{p}_{i j}>\text {. }
$$

Then one determines $\vec{p}_{i j}$ and $\omega(k)$ using eq. (32) and $(35)$, or

$$
k_{j} k_{q} C_{i j p q}^{*} \hat{u}_{p}^{0}-\rho^{*} \omega^{2} \hat{u}_{i}^{0}=0
$$

to which eq. (34) is equivalent on account of eq. (38). As $\vec{p}_{i j}$ and $\omega$ appear implicitly in all quantities, some iterative method is probably required.

3.3 Self-CONSISTENT approximations. - As the exact solution $\omega(k)$ is independent of the auxiliary parameters $C^{0}$, their values are thus far chosen only for mathematical convenience. If, however, one makes approximations, the error will in general depend on $C^{0}$. The reason $C^{0}$ was introduced to begin with was to use it to optimize a given approximation. As these are inevitably made in the integral, it seems a good idea to select $C^{0}$ such that $\hat{p}_{i j}(x)$ fluctuates around zero. From the condition

$$
<\hat{p}_{i j}(x)>=0
$$

one has according to eq. (39)

$$
C^{*}=C^{0}
$$

and then, of course

$$
\rho^{*}=\rho^{0} .
$$

Such a choice is called "self-consistent". In the language of multiple scattering theory this is equivalent to the vanishing of the average $T$-matrix

$$
<T>=0 \text {, }
$$

which there, too, defines the effective Hamiltonian. As before, the implicit equations can best be solved by an iterative procedure, starting with any $C^{0}, \rho^{0}$, calculating the corresponding approximate $C^{*}, \rho^{*}$, taking these as new $C^{0}, \rho^{0}$, etc. 
3.4 CoHerent Potential approximation. - An approximation involving only trivial mathematics is obtained by «neglecting» the integral in eq. (29). This gives, in tensor notation :

$$
\hat{p}(x)+\varphi(x)\left[r<\hat{p}>-\hat{\varepsilon}^{0}\right]=0 .
$$

The space average is

$$
\left\langle\hat{p}>+\left\langle\varphi>\left[\Gamma<\hat{p}>-\hat{\varepsilon}^{0}\right]=0\right.\right.
$$

or

$$
<\hat{p}>=[1+<\varphi>r]^{-1}<\varphi>\hat{\varepsilon}^{0} .
$$

Substitution in eq. (39) gives the approximate value of $C^{*}$, which is obviously dependent on $C^{0}$. For $\rho^{*}$ in this approximation one must take, as shown below :

$$
\rho^{*}=\langle\rho(x)\rangle .
$$

This approximation is made self-consistent by requiring

$$
<\varphi(x)>=0
$$

and of course $\rho^{0}=\langle\rho(x)\rangle \equiv \rho^{*}$. One sees from eq. (29) that in this case the neglected term contains the product of two "random » variables, both fluctuating around zero.

Eq. (47) and (39) suggest an iterative procedure to satisfy eq. (49) : with some initial $C^{0}$, eq. (47) gives $\langle\hat{p}\rangle$ which, in eq. (39), gives an approximation to $C^{*}$, which is used as the next $C^{0}$ in eq. (47), etc. In the end, one arrives at an approximation for $C^{*}$, which I call $C^{* 0}$. Likewise, I call $\langle\rho(x)\rangle=\rho^{* 0}$. $C^{* 0}$ depends on $\omega$ but not on $k$. The approximation to the dispersion relation $\omega(k)$ is obtained from eq. (40) with these values.

One may not think much of this approximation, which avoids all the hard work, but its analogue in the resolvent formalism for the quantum mechanical wave equation is the coherent potential approximation (CPA) (single site approximation), which is about all one has thus far used. The analogy is seen by taking the space average of eq. (29) : neglecting the integral is equivalent to replacing the average of $\varphi(x)$ times a factor involving $p\left(x^{\prime}\right)$ or $u\left(x^{\prime}\right)$ in a different point $x^{\prime}$ by the product of the averages, which gives zero on account of the form of the second factor. The same truncation, used in eq. (36), gives

$$
\rho^{*}=\langle\rho(x)\rangle \text {. }
$$

All improvements on this contain the correlation between the properties in different points of the medium and thus go beyond the CPA. This extremely simple form of the CPA is the basis of my earlier statement that the classical wave equation is much easier to handle than Schrödinger's equation.

3.5 Self-CONSISTENT imBeDding. - It has been shown [4] that the dispersion function of a hetero- geneous wave equation is a proximity property, i.e., depends only on the form of the wave operator in small neighborhoods.

Eq. (29) is well suited to yield approximate solutions based on this property. To this end I consider an equation just like (29), but with the medium $C(x)$, $\rho(x)$ replaced by a uniform medium, except in a small volume $\Omega$, where it is left unchanged. I aim to take as this medium the effective medium, but considering $C^{0}, \rho^{0}$ as some zero-order approximation to it, I imbed first in the medium $C^{0}, \rho^{0}$, which is also used to define $p, \varphi, S$ and $T$. One could e. g. take $C^{0}=C^{* 0}$, $\rho^{0}=\rho^{* 0}$.

Thus one has, using a label $\Omega$ and omitting the indices,

$$
\begin{aligned}
& \varphi^{\Omega}(x)=\varphi(x), \quad x \in \Omega \\
& \varphi^{\Omega}(x)=0, \quad x \notin \Omega
\end{aligned}
$$

and therefore, from eq. (29) and in accordance with the definition (16)

$$
\hat{p}^{\Omega}(x)=0, \quad x \notin \Omega
$$

while one expects

$$
\hat{p}^{\Omega}(x) \approx \hat{p}(x), \quad x \in \Omega .
$$

The integral of the modified eq. (29) is to be extended over $\Omega$ only, and $\bar{p} \equiv\langle\hat{p}(x)\rangle$ is kept as a constant to be determined.

The solution $\hat{p}^{\Omega}(x)$ and $\hat{u}^{\Omega}(x)$ (inside $\Omega$ ) of this equation and of eq. (16) is now determined for an ensemble of neighborhoods $\Omega$. Then I impose the condition

$$
<\hat{p}\rangle=\left\langle<\hat{p}^{\Omega}(x)\right\rangle_{\Omega}>_{\mathrm{av}},
$$

where $<>_{\Omega}$ means average over $\Omega$ and $<>_{\mathrm{av}}$ means ensemble average.

Having satisfied eq. (53) the now well-defined solution $\hat{u}^{\Omega}(x)$ is used to identify and calculate

$$
\langle\rho \hat{u}\rangle=\left\langle<\rho(x) \hat{u}^{\Omega}(x)>_{\Omega}\right\rangle_{\mathrm{av}} .
$$

One can also calculate, by a similar identification, $\langle\hat{u}\rangle$ and $\langle\hat{\varepsilon}\rangle$, and expects of course to find values near $\hat{u}^{0}$ and $\hat{\varepsilon}^{0}$. Finally, $C^{*}$ and $\rho^{*}$ are found from eq. (39) and (36).

Due to the imbedding, this is only an approximate value. All of this must be done as a function of $\omega$, at least for $\omega^{\prime} s$ in the vicinity of the expected solution $\omega(k)$. With this « first " approximation for $C^{*}$ and $\rho^{*}$, one can solve the eigenvalue equation of $(40)$ to find the first approximation to $\omega$.

Next, one takes these approximate values of $C^{*}$, $\rho^{*}$ as new $C^{0}$ and $\rho^{0}$ and repeats the entire calculation. One expects a smaller error, because one imbeds in a medium more like the effective medium. On also redetermines $\omega$, etc. When this iteration procedure converges, one obtains an approximation to $C^{*}$, $\rho^{*}$ corresponding to an estimated value $\langle\hat{p}\rangle=0$. 
The errors due to imbedding depend on the size of $\Omega$, and in the limit $\Omega \rightarrow \infty$ the theory becomes exact.

3.6 LONG WAVELENGTH OR SMALL DENSITY CONTRAST. - The whole theory would be much simpler without the second term in the integral of eq. (29), containing $u_{i}(x)$. This term is small of the order of "grain" diameter-to wavelength ratio compared to the leading part of the first term, and is obviously unimportant when $\rho(x)$ is nearly constant, whatever be the wavelength or variations of $C(x)$. For the case that one wants to treat this term as a perturbation, I will briefly discuss the simplified equation obtained by dropping it. One obtains now an equation for $p(x)$ only. $C^{*}$ follows from eq. (39) and $\rho^{*}$ is the space-average of $\rho(x)$, i. e. $\rho^{* 0}$. Introducing a tensor $q(x)$ by

$$
\widehat{p}(x)=q(x) \hat{\varepsilon}^{0},
$$

one has

$$
\begin{gathered}
q(x)+\varphi(x) \int d^{3} x^{\prime} \hat{T}\left(x-x^{\prime}\right)\left(q\left(x^{\prime}\right)-<q>\right)+ \\
+\varphi(x)(\Gamma<q>-1)=0
\end{gathered}
$$

where $" 1 »$ is the unit tensor as in eq. (27).

$C^{*}$ is given by

$$
C^{*}=C^{0}+\langle q\rangle
$$

and $\omega(k)$ follows from

$$
\operatorname{Det}\left(k_{j} k_{q} C_{i j p q}^{*}-\rho^{* 0} \omega^{2} \delta_{i p}\right)=0 .
$$

Equations of the form (56) and (57) have been derived earlier [5] for the limit of static deformations. The only difference is that $T$ and $\Gamma$ (and therefore $\varphi$ ) obtained in reference [5] are the long-wavelength and zero frequency limit of $\widehat{T}$ and $\Gamma$ of eq. (56). As a consequence of this similarity in form one can, beside imbedding methods, also use an approximation based on the calculation of 2- and 3-point correlation functions that was developed in reference [5] for the static case. And, of course, imbedding calculations are greatly simplified. It is unfortunate though inescapable that strong fluctuations in the density will cause serious discrepancies between this approximation and reality.

4. Epilogue. - I hope to have shown that it pays to transform the wave equation before going ahead with approximation methods. The transformations used contain the following essential ingredients. 1) With boundary conditions as for a plane wave displacement field, the wave equation is written as an integral equation. 2) A more singular kernel is obtained by partial integration and differentiation. This seemingly enhances the short-range contributions, thus favoring the use of the proximity property. 3) As a consequence, the integral becomes the principal value, after yielding a finite contribution at the singular point. The importance of this lies in the fact that the CPA is now obtained by neglecting the integral. 4) Taking the limit for an infinite volume produces a term proportional to the mean stress. This term disappears when the model-medium is chosen self-consistently, but plays an important role in the convergence of iteration methods. 5) As a last but decisive step the equation is written as an integral equation for the stress instead of the strain. This changes completely any result based on averaging procedures and also yields, in a very natural way, a self-consistent imbedding approximation based on the proximity property.

In the extensive literature on the classical random wave equation [6] the points 2)-5) have, to my knowledge, never been emphasized. Still, they seem to simplify the use of statistical methods, although the correlation functions are of an entirely different type than usual. To what extent these transformations are also useful for a Schrödinger-type wave equation remains to be investigated.

Acknowledgment. - The author is indebted to Dr R. L. Mills for valuable discussions.

\section{References}

[1] See for example Messiah A., Quantum Mechanics [North-Holland Publ. Co., Amsterdam] (1963), 712.

[2] Soven P., Phys. Rev. 156 (1967) 809.

[3] Freed K. F. and Cohen M. H., Phys. Rev. B 3 (1971) 3400 .

Vanderbauwhede A. and Phariseau P., Physica 56 (1971) 379 and several recent papers by other authors.
[4] Butler W. H. and KoHN W., Local Theory of Disordered Systems. Electronic Density of States Conference, 1969, NBS Special Publication \# 323 (1971), 465.

[5] Korringa J., J. Math. Phys. to be published.

[6] Frisch U., Wave Propagation in Random Media, Probabilistic Methods in Applied Mathematics, vol. 1, 75-198, 1968 [Acad. Press, Ed. A. T. Bharucha-Reid]. In this paper one finds 156 further references. 